\title{
Low Cost Electrochemical Sensor for Simultaneous Detection and Estimation of Dihydroxybenzene Isomers
}

\author{
N.T. Tonu ${ }^{1,2}$ and M.A. Yousuf ${ }^{1, *}$ \\ ${ }^{1}$ Department of Chemistry, Khulna University of Engineering \& Technology, \\ Khulna-9203, Bangladesh \\ ${ }^{2}$ Chemistry Discipline, Khulna University, Khulna-9208, Bangladesh \\ "Corresponding author: yousuf@chem.kuet.ac.bd \\ Received 02/06/2019; accepted 15/06/2021 \\ https://doi.org/10.4152/pea.2022400105
}

\begin{abstract}
An ionic liquid (IL) based pencil graphite electrode (PGE) sensor was electrochemically fabricated for hydroquinone (HQ), catechol (CC) and resorcinol (RS) simultaneous detection and estimation in aqueous media. PGE surface was modified by 1-hexylpyridinium hexafluorophosphate (HPHP) using cyclic voltammetry (CV). The modified surface was characterized by scanning electron microscope (SEM) and energy dispersive X-ray microanalysis (EDX). The modified electrode showed an excellent electro-analytical activity towards simultaneously $\mathrm{HQ}, \mathrm{CC}$ and RS, at pH 6.8 in aqueous media. The scan rate effect was diffusion controlled and the concentration effect was linear with current. The limit of detection (LOD) for HQ, CC and RS was found to be $6.38 \mu \mathrm{mol} \mathrm{L}{ }^{1}, 4.56 \mu \mathrm{mol} \mathrm{L}^{1}$ and $19.6 \mu \mathrm{mol} \mathrm{L}{ }^{1}$, respectively. The sensitivity for HQ, CC and RS was found to be $448.49 \mu \mathrm{AmM}^{-1} \mathrm{~cm}^{-2}, 627.35 \mu \mathrm{AmM}^{-1} \mathrm{~cm}^{-2}$ and $146.10 \mu \mathrm{AmM}^{-1} \mathrm{~cm}^{-2}$, respectively, in a ternary mixture of dihydroxybenzene isomers (DHBIs). The cost of using PGE was lower than that of the conventional electrodes.
\end{abstract}

Keywords: Dihydroxybenzene isomers, ionic liquid, pencil graphite electrode and differential pulse voltammetry.

\section{Introduction}

DHBIs (HQ, CC and RS) have a remarkable role in many fields, such as cosmetics, dye, medicines, antioxidant, flavoring agents, tanning, photography chemicals, pharmaceutical and pesticide industries [1]. However, due to their high toxicity and low degradability in the environment, they have been certified as environmental pollutants [2]. Because DHBIs can be absorbed through oral, dermal or respiratory tracts, there are many health problems caused by them [3]. HQ or CC absorption through the gastrointestinal tract can cause renal tube degeneration and liver injury. RS high amounts inhalation can be lethal to human beings [4]. If we see DHBIs chemical structure, we find out that HQ, CC and RS have similar structures and properties and a tendency to coexist in certain products [5]. So, a remarkably and discriminatory analytical method is crucial for DHBIs detection.

Until now, numerous methods have been tried for DHBIs determination, such as high performance liquid chromatography (HPLC) [6], electro- 
chemiluminescence [7], gas chromatography [8], capillary electrochromatography [9], $\mathrm{pH}$ based flow-injection analysis [10] and electrochemical methods [11]. Due to their low cost, high sensitivity and selectivity, electrochemical methods have been used in most of the studies on DHBIs simultaneous detection, and there has been an extra attention given to HQ and CC simultaneous determination, because their redox peaks are overlapped at conventional electrodes [2]. Lately, a number of innovative material-based electrochemical sensors have been developed for DHBIs simultaneous detection, such as graphene doped carbon ionic liquid electrodes [2], ruthenium red/carbon nanotubes [5], modified glassy carbon electrodes based on multiwall carbon nanotubes [11], silver and graphene nanoparticles [12], flower-like Pd-graphene nanocomposites [13], nitrogen doped porous carbon nanopolyhedrons-multiwall carbon nanotubes hybrid materials [14], bamboo-shaped nitrogen-doped carbon nanotubes [15], cadmium sulphide/reduced graphene oxide sandwiched films [16], aminated glassy carbon electrode [17], meso-porous platinum electrodes [18], carbon nanofibers and gold nanoparticles modified gold electrodes [19], micro sensors based on poly (3,4-ethylenedioxythiophene) modified carbon fiber electrodes [20], etc.

Until now, researchers have used expensive chemicals and electrodes, such as multi-walled carbon nanotubes which cost around $€ 100 /$ gram, silver nanoparticles which cost $€ 800 /$ gram, graphene which costs $€ 650 /$ gram [Sigma-Aldrich], glassy carbon electrodes which cost around $\$ 98-\$ 358 /$ piece, platinum electrodes which cost $\$ 30-95 /$ piece and gold electrodes which cost $\$ 80-\$ 120 /$ piece [Alibaba]. Some researchers have used carbon paste electrodes [2], with an electrical conducting paste made of carbon particles, such as pure graphite or graphene and a binder, such as ionic liquids (IL). IL are organic salts with a low melting point. They are mostly liquid at room temperature. On account of their exclusive properties, such as non-volatility, low toxicity, biocompatibility, high thermal conductivity and stability, IL have been used in electrochemical sensors [21]. For example, Li Ma et al. have developed a graphene doped carbon ionic liquid electrode for HQ, CC and RS simultaneous detection [2]. Xiaoying Sun et al. have fabricated a carbon ionogel electrode which was based on butyl-3methylimidazolium hexafluorophosphate for HQ electrochemical detection [22]. Sheying Dong et al. have fabricated a hydrophobic ionic liquid functionalized SBA-15 modified carbon paste electrode [23].

Graphite powder is inexpensive and it is very easy to make a carbon paste with it. But the main problem of a carbon paste electrode is that it needs special methods to be activated, maintained and conserved. A graphite soft rod, such as a graphite pencil, can be used instead of powder, as the chemical binder has a tendency to penetrate amongst the graphite rod particles or to make a thin film on its working surface.

In modern electroanalytical field, PGE has been brilliantly stood in as a sensor. It is a combination of graphite particles, polymeric binders, additives, such as clay, 
and it is a porous composite. Because of its low cost, good mechanical rigidity, high electrochemical activity, renewable surface and simplicity of modification, it has been used in metal ions detection. In order to have a large surface area, PGE has the ability to detect low analyte concentrations. This category of electrode has been fruitfully applied to fabricate several sensors [24]. For example, Arehalli Sathisha et al. have fabricated a poly (glycine) film coated PGE for paracetamol determination [24], and Levent Ozcan et al. have made a molecularly imprinted polypyrrole-modified GPE for ascorbic acid determination [25].

In our study, a low cost, easily modified electrochemical sensor was fabricated with the help of HPHP and ecofriendly PGE which is quite disposable. The thin film on the PGE surface was characterized by micro analytical technology. Using this sensor, a simple and fast evaluation system was assembled for HQ, CC and RS simultaneous detection, with the help of $\mathrm{CV}$ and Differential Pulse Voltammetry (DPV).

\section{Materials and methods}

The chemicals used in this experiment are: (i) hydroquinone (BDH, UK); (ii) catechol (BDH, UK); (iii) resorcinol (BDH, UK); (iv) sodium dihydrogen phosphate (Sigma-Aldrich); (v) disodium hydrogen phosphate (Sigma-Aldrich); (vi) acetic acid (Sigma-Aldrich); (vii) sodium acetate (Sigma-Aldrich); (viii) sodium bicarbonate (Sigma-Aldrich); (ix) sodium hydroxide pellets (SigmaAldrich); and (x) 1-Hexylpyridinium hexafluorophosphate (TCI, Japan). Double distilled water was used for the solutions preparation and apparatus cleaning. The solutions were purged with $99.99 \%$ dry nitrogen (BOC, Bangladesh) to remove oxygen, and the atmosphere was maintained inert prior to investigation. All reagents were obtained as AR grade and used without further refinement. The whole experiment was carried out using a potentiostat ( $\mu$-stat 8000, DropSens, Spain). PGE was used as working electrode, while $\mathrm{Ag}|\mathrm{AgCl}| \mathrm{Cl}^{-}$and a platinum $(\mathrm{Pt})$ wire coil were used as reference and counter electrodes, respectively. The solution $\mathrm{pH}$ was measured using a $\mathrm{pH}$ meter (Hanna Instruments).

\section{IL-PGE preparation}

0.2 M PBS ( $\mathrm{pH}=6.8$ ) was prepared. $0.1 \mathrm{~g}$ HPHP was taken in $40 \mathrm{~mL}$ PBS. Then, the solution was sonicated and stirred until HPHP was mixed with PBS, resulting in a IL-PBS solution. A pencil (Faber Castell, grade 2B) was cut, and the wooden part of the two sides was removed. Then, it was painted, but the end tip of the rod remained unpainted for using it as a circular electrode surface ( $2 \mathrm{~mm}$ diameter). The side portion of the graphite rod was painted, so that only the uncovered portion could make part of the working surface. Then, the working surface was polished by rubbing it on a smooth offset white paper. The resultant working surface looked like a shiny black mirror. The unpainted part was used to make the connection with the potentiostat.

The bare PGE was rinsed with distilled water. Then, it was placed in an IL-PBS solution which was previously purged with highly pure nitrogen for $5 \mathrm{~min}$. The PGE was treated with 15 cycles of $\mathrm{CV}$ from -1.0 to $+1.7 \mathrm{~V}$, at a scan rate of 
$300 \mathrm{mV}$. Then, the modified electrode was rinsed with double distilled water for further experiments.

\section{IL-PGE morphological characterization}

Fig. 1(a) shows the bare PGE surface morphology. The greyish-black color corresponds to the graphite on the surface that was also uneven and had a lot of grooves. This indicates that the pencil graphite rod is not purely crystalline, and that several defects and a few foreign materials may be present there. Fig. 1(b) shows the modified IL-PGE SEM images. The modified electrodes surface images are different from those of the bare PGE. The IL-PGE surface is covered by many white spots and fibrous lines of different sizes, which may be thin IL films spread over the PGE surface. This modified PGE can be expected to be an attractive platform for the DHBIs detection and separation, and used to construct very effective electrochemical sensors.
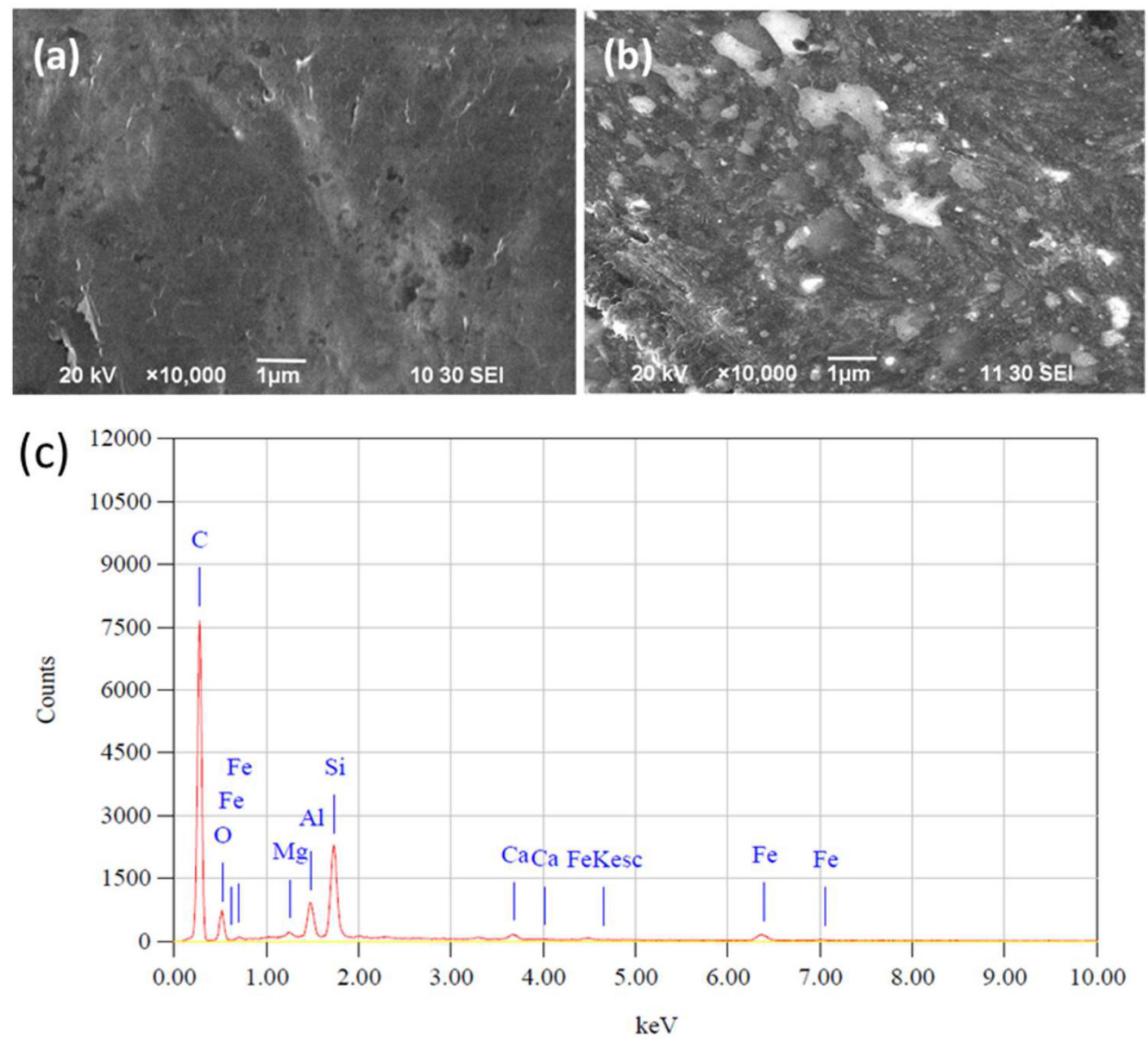

Figure 1. SEM image of (a) bare PGE, (b) IL-PGE and (c) bare PGE EDX.

Fig. 1(c) shows the bare PGE surface EDX results. The PGE surface is impure, as analyzed by SEM. It is composed of $79.39 \%$ carbon, $10.03 \% \mathrm{Si}, 3.06 \% \mathrm{O}_{2}$, $3.49 \% \mathrm{Al}, 2.68 \% \mathrm{Fe}$ and a trace amount of $\mathrm{Mg}$ and $\mathrm{Ca}$. In the bare GCE, there is $100 \%$ carbon without impurities. So, it may be concluded that PGE is a mainly carbon composite material. In spite of the impurities present in the graphite of 
PGE, it has been successfully modified by IL, and exhibits a noble response for the simultaneous DHBIs detection.

\section{Results and discussion}

\section{DHBIs behavior at bare and modified PGE}

Fig. 2 shows HQ, CC and RS CVs at the bare PGE and IL-PGE, as well as the PBS CVs. It is seen that there is no peak for PBS, for both electrodes, but a small charging current was found. HQ showed one anodic and one cathodic peak, RS showed only one anodic peak and $\mathrm{CC}$ exhibited one anodic and two cathodic peaks at the bare PGE (solid lines in Fig. 2). At IL-PGE, HQ showed one anodic and one cathodic peak, CC showed one anodic peak and one cathodic peak and RS showed only one anodic peak (dashed lines in Fig. 2). At IL-PGE, HQ, CC and RS showed reversible, quasi-reversible and irreversible behavior, respectively. It is clear from CVs that bare PGE gave broader and confusing peaks, while IL-PGE provided relatively more selective and specific peaks.

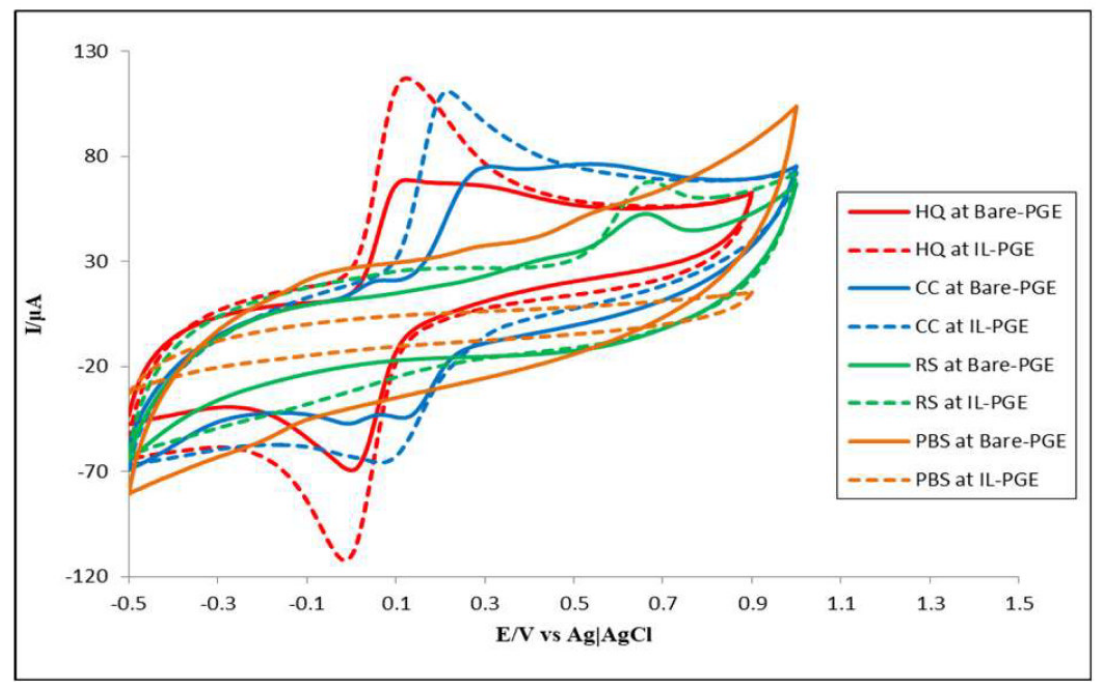

Figure 2. CVs of $5 \mathrm{mM} \mathrm{HQ}, \mathrm{CC}$ and RS, at bare-PGE (solid lines) and at IL-PGE (dotted lines) in PBS.

\section{pH effect}

$\mathrm{HQ}, \mathrm{CC}$ and RS activity was examined by taking the experiments in acetate, phosphate and bicarbonate buffers. $\mathrm{pH}$ ranged from 3 to 11. HQ, CC and RS gave sharp peaks at $\mathrm{pH} 6-7$, which was thus considered the optimum $\mathrm{pH}$. Similar experiments were also carried out in PBS; $\mathrm{pH}$ ranged from 5.8 - 7.2. HQ gave sharp peaks at $\mathrm{pH}$ 6.8, which was thus considered the optimum $\mathrm{pH}$.

\section{DHBIs binary mixtures electrochemical behavior at IL-PGE}

A succession of $\mathrm{CV}$ and DPV experiments were performed on binary DHBIs mixtures, at $\mathrm{pH} 6.8$, for their simultaneous detection.

\section{$H Q$ and $C C$ mixture detection in PBS}

$\mathrm{HQ}$ and CC exhibited well separated anodic peaks, at $+110 \mathrm{mV}$ and $+155 \mathrm{mV}$, and two cathodic peaks, at $+10 \mathrm{mV}$ and $+172 \mathrm{mV}$, respectively. 
A distinct HQ and CC CVs overlay, with their HQ + CC binary mixture (Fig. 3(a)), indicates that they both held their corresponding redox peaks in the ILPGE binary mixture.

A HQ + CC binary mixture DPV in PBS is presented in Fig. 3(b), which undoubtedly confirms CC and HQ responses with well separated anodic peaks, while holding their specific locations at $+5 \mathrm{mV}$ and $+125 \mathrm{mV}$, respectively, at IL-PGE. The peak departure was measured to be $120 \mathrm{mV}$; that is fairly good for the simultaneous detection of these isomers in one another presence.

However, CC peak current is a little higher in its mixture with HQ than when it is alone in PBS. This interference may cause a problem in the $\mathrm{CC}$ quantitative estimation from its mixture with HQ.

\section{CC and RS mixture detection in PBS}

Similar tests were done on CC-RS binary system. In the CVs, CC and RS presented responses with a large peak separation, which was retained in the binary mixture [Fig. 3(c)]. In DPV (Fig. 3(d)), CC and RS showed oxidation peaks (at $+105 \mathrm{mV}$ and $+530 \mathrm{mV}$ ) separated by $425 \mathrm{mV}$; that is suitable for their simultaneous detection and quantification at IL-PGE.

\section{$H Q$ and $R S$ mixture detection in PBS}

Similar experiments were performed on HQ-RS binary system that exhibit an even better peak separation in both CV and DPV represented in Fig. 3(e) and 3(f), respectively. In DPV, the binary mixture exhibits two anodic peaks, $+10 \mathrm{mV}$ and $+535 \mathrm{mV}$, respectively, resulting in a peak separation of $525 \mathrm{mV}$.

The graphite in a pencil is not as pure as the graphite in conventional graphite electrodes. PGE has many other elements, as confirmed by the EDX result. The peaks obtained from CVs could be shifted a little left or right because of two reasons. One is the ohmic drop and another is the electrode fouling effect. Here, the latter is more pronounced than the former. When a potential is applied to the PGE, there could be an obstacle for the electrons transfer through the electrode, because there are gluing substances, used to bind the graphite materials in a pencil, which are electrically non-conducting. So, the graphite distribution in a PGE rod is not homogeneous, resulting in a confusing position of cathodic peaks in Fig. 3a, 3c, 3e and $3 \mathrm{~g}$.

\section{$H Q, C C$ and $R S$ ternary mixture detection in PBS}

Individual HQ, CC and RS CVs and that of their mixture in PBS at IL-PGE are overlaid on Fig. $3(\mathrm{~g})$. Two anodic peaks, at $+308 \mathrm{mv}$ and $+736 \mathrm{mV}$, are observed. The peak at $+736 \mathrm{mV}$ indicates the $\mathrm{RS}$ anodic peak, but the peak at $+308 \mathrm{mV}$ is the HQ and CC overlapped anodic peak. The DPV study of HQ, CC and RS, as individual isomers and as mixture, is shown in Fig. 3(h), which shows that, at IL-PGE, HQ, CC and RS retain their corresponding anodic peaks at $+10 \mathrm{mV}$, $+125 \mathrm{mV}$ and $+535 \mathrm{mV}$, respectively, in their ternary mixture. It is seen that the HQ peak current has got a relatively smaller value in the HQ, CC and RS ternary mixture than that of when it is alone in PBS. This interference may hamper the HQ quantitative calculation from the also earlier discussed ternary mixture with $\mathrm{HQ}$ and $\mathrm{CC}$ in PBS. 

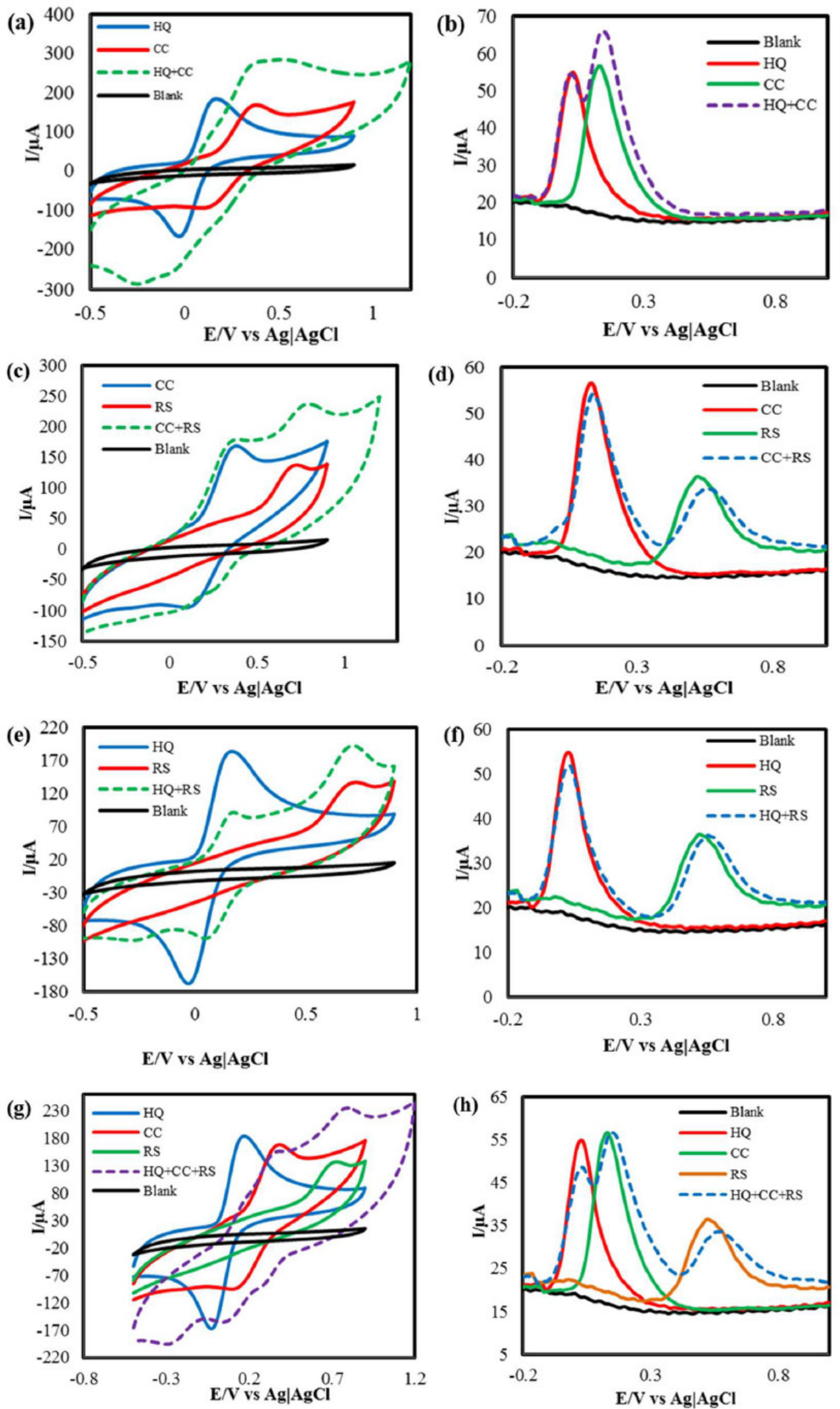

Figure 3. (a) CVs and (b) DPVs of $5 \mathrm{mM}$ of HQ and CC, and of their binary mixture in PBS; (c) CVs and (d) DPVs of $5 \mathrm{mM}$ of $\mathrm{CC}$ and RS, and of their binary mixture in PBS; (e) CVs and (f) DPVs of $5 \mathrm{mM}$ of HQ and RS, and of their binary mixture in PBS; (g) CVs and (h) DPVs of $5 \mathrm{mM}$ of HQ, CC and RS, and of their ternary mixture in PBS. 
The bare PGE surface is not pure graphite. There are many other materials, as confirmed by the EDX result. After every rubbing, a new surface is formed. The new surfaces may have different elements associated with carbon. These extra elements might have an effect on the electricity passes, which causes an electrode fouling. From Fig. 3 (a, c and e), it is seen that the anodic peak potential value shifted towards positive or negative values. The shifting tendency of the $\mathrm{E}_{\mathrm{pa}}$ values, for HQ, CC and RS, results from the electric double layer formation around the electrode surface and from the extra foreign materials present in the latter.

\section{$H Q$ quantitative estimation in $C C$ and $R S$ presence}

DPV was performed on the HQ, CC and RS ternary mixture at IL-PGE, within the potential range from $-0.2 \mathrm{~V}$ to $+1.0 \mathrm{~V}$. A ternary solution was prepared, where $\mathrm{CC}$ and $\mathrm{RS}$ were kept at a constant concentration of $3 \mathrm{mM}$, and the HQ concentration was increased by adding successive amounts of it in the ternary solution, every time. The resulting DPVs are shown in Fig. 4(a).
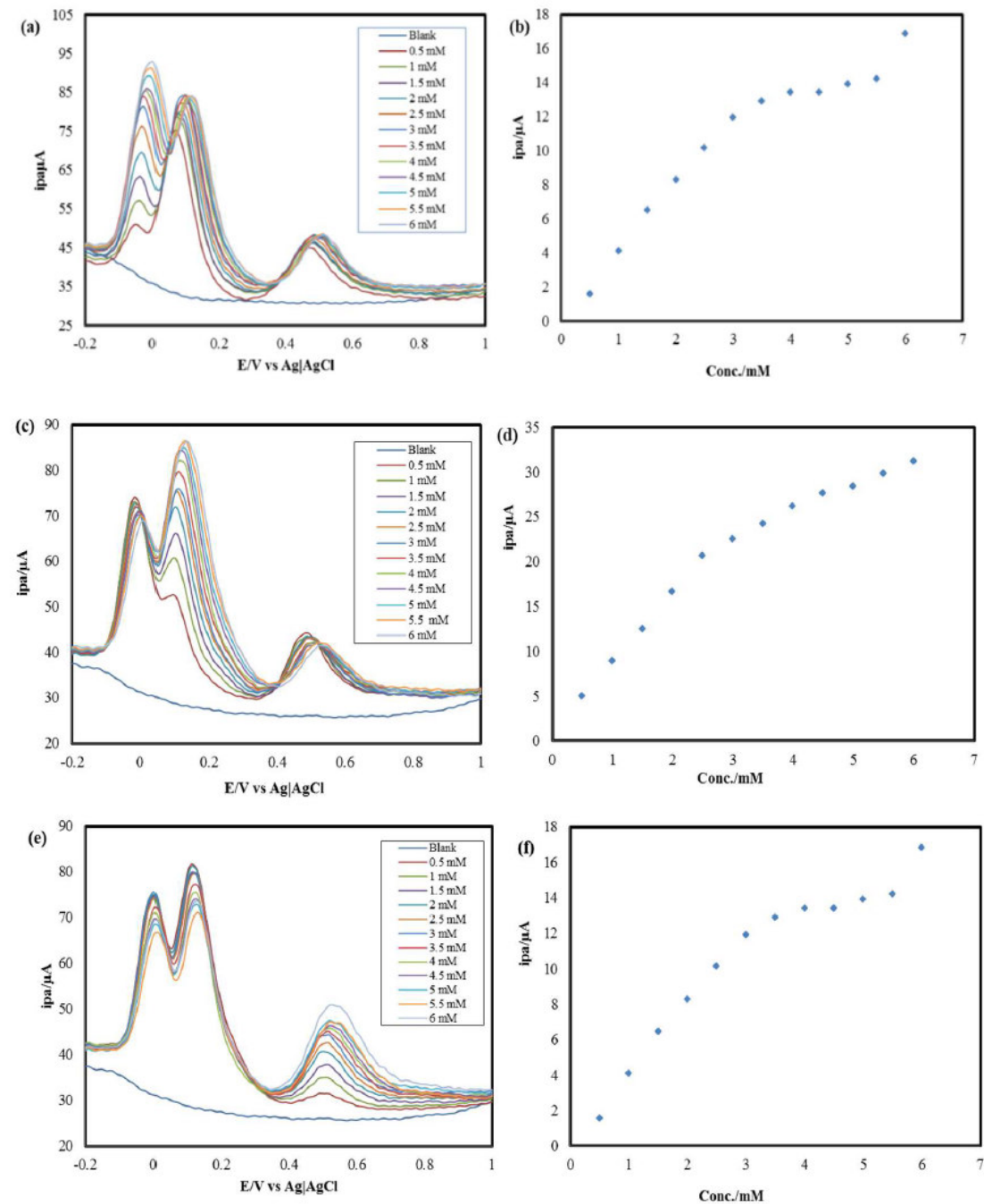

Figure 4. DPVs of (a) HQ in CC and RS presence, (b) HQ in a ternary mixture with CC and $\mathrm{RS}$, (c) $\mathrm{CC}$ in HQ and RS presence, (d) $\mathrm{CC}$ in a ternary mixture with HQ and RS (e) $\mathrm{RS}$ in HQ and $\mathrm{CC}$ presence, at IL-PGE, in PBS. Calibration curve for the quantitative determination of (f) RS in a ternary mixture with HQ and CC. 
A calibration curve [Fig. 4(b)] was drawn for different HQ concentrations in its ternary mixture with $\mathrm{CC}$ and $\mathrm{RS}$. The HQ detection limit in $\mathrm{CC}$ and $\mathrm{RS}$ presence was found in a micromolar range.

\section{CC quantitative estimation in $H Q$ and $R S$ presence}

DPV was performed on the HQ, CC and RS ternary mixture, at IL-PGE, within the potential range from $-0.2 \mathrm{~V}$ to $+1.0 \mathrm{~V}$. A ternary solution was prepared, where HQ and RS were kept at a constant concentration of $3 \mathrm{mM}$, and the CC concentration was increased by adding successive amounts of it in the ternary solution, every time. The resulting DPVs are shown in Fig. 4(c). A calibration curve [Fig. 4(d)] was drawn for different CC concentrations in its ternary mixture with HQ and RS. The CC detection limit in HQ and RS presence was found in a micromolar range.

\section{$R S$ quantitative estimation in $H Q$ and $C C$ presence}

DPV was performed on the HQ, CC and RS ternary mixture, at IL-PGE, within the potential range from $-0.2 \mathrm{~V}$ to $+1.0 \mathrm{~V}$. A ternary solution was prepared, where HQ and CC were kept at a constant concentration of $3 \mathrm{mM}$, and the RS concentration was increased by adding successive amounts of it in the ternary solution, every time. The resulting DPVs are shown in Fig. 4(e). A calibration curve [Fig. 4(f)] was drawn for different RS concentrations in its ternary mixture with CC and HQ. The RS detection limit in CC and HQ presence was found in a micro-molar range. This separating ability of the IL-PGE can be used to quantitatively estimate $\mathrm{HQ}, \mathrm{CC}$ and RS in each other's presence.

\section{Probable mechanism for the DHBIs separation}

HPHP has a bulky 1-Hexylpyridinium cation that contains a six membered alkyl chain with hydrogen atoms at its end. Graphite in PGE has a sea of electrons at its surface. When a potential is applied to PGE, the electropositive hydrogen atoms get attracted to the PGE surface electrons and form a weak bond, resulting in an IL-PGE surface [Fig. 5] that acts as an electro-active surface for DHBIs.
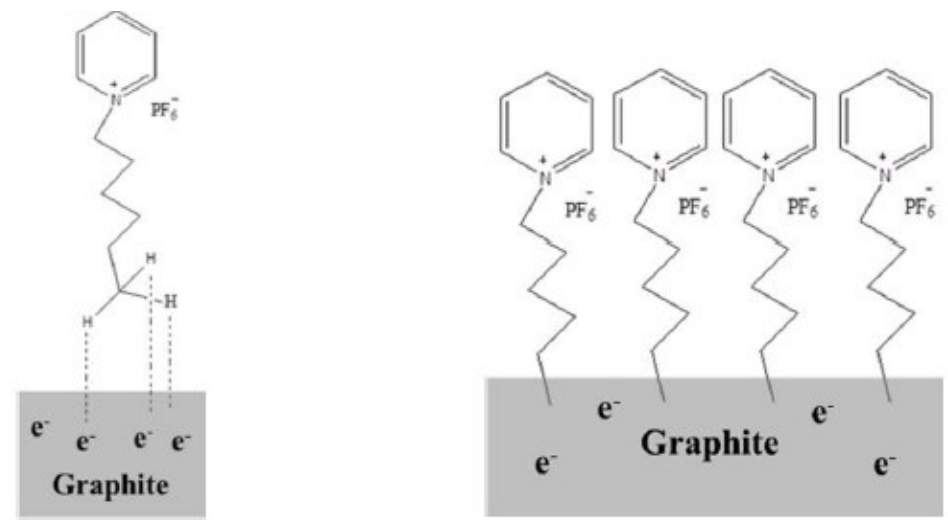

Figure 5. Weak bond formation between the PGE surface and HPHP (left) and the modified IL-PGE surface (right).

From the CVs results, it has been seen that, in their redox reactions, HQ is reversible, $\mathrm{CC}$ is quasi-reversible and RS is irreversible (Fig. 6). Therefore, the possible mechanisms might be shown as Fig. 7. 


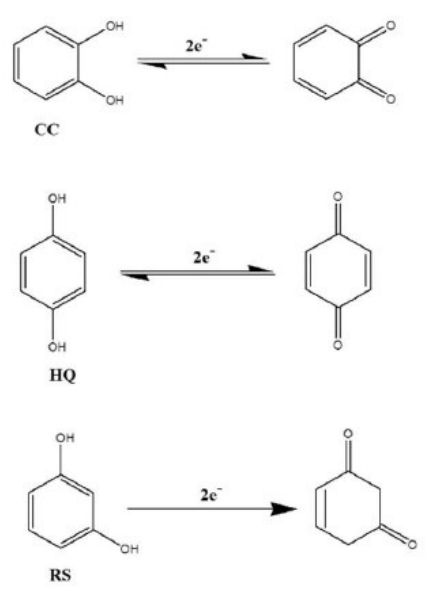

Figure 6. DHBIs electrochemical behavior.
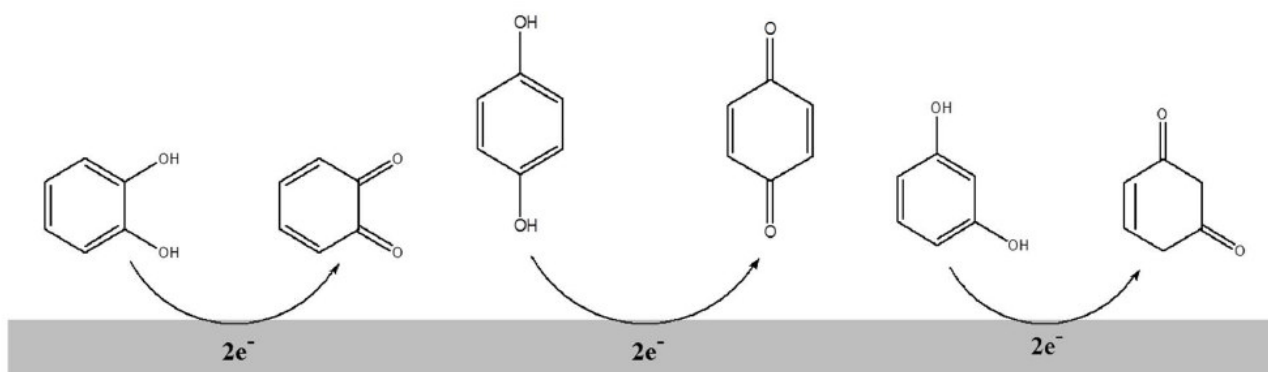

IL-PGE

Figure 7. DHBIs reaction mechanism at the IL-PGE surface.

\section{IL-PGE novelty}

Conventional electrodes are not much available and they are greatly expensive, while wooden pencil is available in the market and, by using PGE, the cost can be reduced about two thousand times, as compared to conventional electrodes. The IL-PBS solution was taken to modify glassy carbon, platinum and gold electrodes, according to the procedure above described for the PGE modification. After PGE was supposedly modified to IL-PGE, it did not show satisfactory results. Here, two things could have happened: either the modification did not happen or it happened, but its platform did not act in a favorable way for the DHBIs electrochemical behavior.

\section{Electrochemical results reproducibility and feasibility}

A reproducibility test has been performed for electrochemical results. Sixty ILPGEs sensors have been fabricated during the research work. From the test, it was seen that each electrode provided almost similar electrochemical results until eight successive experiments, but, after that, the peak current gradually decreased. Their reproducibility was investigated by comparing the DPV peak current of $1 \mathrm{mM} \mathrm{HQ}, \mathrm{CC}$ and RS ternary mixtures. After eight experiments, the relative standard deviation of five repeated experiments was $3.59 \%$ for HQ, $1.58 \%$ for $\mathrm{CC}$ and $3.14 \%$ for RS, indicating an acceptable reproducibility. The electrode stability has been also tested. Ten sensors used in the above described investigation were stored in an empty covered beaker for 4 weeks. The results 
showed that the peak current retained $\sim 95 \%$ of its initial values, revealing the sensor stability.

\section{Conclusion}

A cost-effective and simple electrochemical sensor was fabricated by modifying PG for simultaneous HQ, CC and RS detection and estimation, from their binary and ternary mixtures. PG was modified in a HPHP-PBS solution, by using CV for fabricating PGE. CV and DPV techniques were used for simultaneous DHBIs detection and quantification. HQ, CC and RS have been detected from their binary and ternary mixtures, with sensitivity and selectivity at IL-PGE, although there is a $\mathrm{CC}$ estimation limit in the $\mathrm{HQ}+\mathrm{CC}$ binary mixture from the DHBIs ternary mixture. Modified PGE is at least 2000 times cheaper than conventional GCE.

\section{References}

1. Selvakumar P, Chelladurai K, Shen-Ming C, et al. Simultaneous and selective electrochemical determination of Dihydroxybenzene isomers at a reduced graphene oxide and copper nanoparticles composite modified glassy carbon electrode. Analyt Methods. 2014;6:4271-4278. https://doi.org/10.1039/C4AY00433G

2. Li M, Guang-Chao Z. Simultaneous determination of hydroquinone, catechol and resorcinol at graphene doped carbon ionic liquid electrode. Int $\mathbf{J}$ Electrochem. 2012;2012:1-8. https://doi.org/10.1155/2012/243031

3. Su-Juan L, Yun X, De-Hua D, et al. A comparative study of different types of reduced graphene oxides as electrochemical sensing platforms for hydroquinone and catechol. J Solid State Electrochem. 2015;19:861-870. http://dx.doi.org/10.1007\%2Fs10008-014-2692-8

4. Lv M, Wei M, Rong F, et al. Electrochemical detection of catechol based on As-grown and nanograss array boron-doped diamond electrodes. Electroanalysis. 2010;22:199-203. https://doi.org/10.1002/elan.200900296

5. Mohammad MF, Meissam N, Mozhgan KM. Simultaneous determination of hydroquinone and catechol using a modified glassy carbon electrode by ruthenium red/carbon nanotube. J Iran Chem Soc. 2015;12:1-9. http://dx.doi.org/10.1007/s13738-014-0575-7

6. Lin $\mathrm{CH}$, Sheu JY, Wu HL, et al. Determination of hydroquinone in cosmic emulsion using microdialysis sampling coupled with high-performance liquid chromatography. J Pharm and Biomed Anal. 2005;38:414-419. https://doi.org/10.1016/j.jpba.2005.01.031

7. Li SF, Li XZ, Xu J, et al. Flow-injection chemiluminescence determination of polyphenols using luminol-NaIO -gold nanoparticles system. Talanta. 2008;75:32-37. https://doi.org/10.1016/j.talanta.2007.10.001

8. Moldoveanu SC, Kiser M. Gas chromatography/mass spectrometry versus liquid chromatography/fluorescence detection in the analysis of phenols in mainstream cigarette smoke. J Chromatogr A. 2007;1141:90-97. https://doi.org/10.1016/j.chroma.2006.11.100

9. Guan N, Zeng Z, Wang Y, et al. Open tubular capillary electrochromatography in fused-silica capillaries chemically bonded with 
macrocyclic dioxopolyamine. Analyt Chim Acta. 2000;418:145-151. http://dx.doi.org/10.1016/S0003-2670(00)00951-X

10. Garcia-Mesa JA, Mateos R. Direct automaticdeterination of bitterness and total phenolic compounds in virgin oliv oil using a $\mathrm{pH}$-based flow-injection analysis system. J Agric Food Chem. 2007;55:3863-3868. http://dx.doi.org/10.1021/jf070235v

11. Yan-ping $\mathrm{C}, \mathrm{Yu} \mathrm{Z}, \mathrm{Yi}$-lian $\mathrm{L}$, et al. Electrochemical behavior of Dihydroxybenzene isomers at MWCNTs modified electrode and simultaneous determination in neutral condition. Res Chem Intermed. 2014;40:1-10. http://dx.doi.org/10.1007/s11164-013-1161-9

12. Ke-Jing H, Lan W, Jing L, et al. Electrochemical sensing of catechol using a glassy carbon electrode modified with a composite made from silver nanoparticles, polydopamine and graphene. Microchim Acta. 2013;180:1-7. https://doi.org/10.1007/s00604-013-0988-5

13. Min Z, Feng G, Faling C. Preparation of flower-like Pd-graphene coposites for simultaneous determination of catechol and hydroquinone. Res Chem Intermed. 2015:1-14. https://doi.org/10.1007/s11164-015-2056-8

14. Wei L, Liang $\mathrm{W}$, Xiaohua $Z$, et al. Simultaneous electrochemical determination of hydroquinone, catechol and resorcinol at nitrogen doped porous carbon nanopolyhedrons-multiwall carbon nanotubes hybrid materials modified glassy carbon electrode. Bull Korean Chem Soc. 2014;35:204-210. http://dx.doi.org/10.5012/bkcs.2014.35.1.204

15. Dong J, Qu X, Wang L, et al. Electrochemistry of nitrogen-doped carbon nanotube $\left(\mathrm{CN}_{\mathrm{x}}\right)$ with different nitrogen content and its application in simultaneous determination of Dihydroxybenzene isomers. Electroanalysis. 2008;20:1981-1986. http://dx.doi.org/10.1002/elan.200804274

16. Shirong $H$, Wuxiang $Z$, Jianzhong $Z$, et al. One step cadmium sulphide/reduced graphene oxide sandwiched film modified electrode for simultaneous electrochemical determination of hydroquinone, catechol and resorcinol. RSC Adv. 2015;5:18615-18621. https://doi.org/10.1039/C4RA16268D

17. Xiuyun W, Min X, Mengmeng G, et al. An electrochemically aminated glassy carbon electrode for simultaneous determination of hydroquinone and catechol. Analyst. 2016;141:1-6. https://doi.org/10.1039/C5AN02098K

18. Ghanem MA. Electrocatalytic activity and simultaneous determination of catechol and hydroquinone at mesoporous platinum electrode. Electrochem Communic. 2007;9:2501-2506. https://doi.org/10.1016/j.elecom.2007.07.023

19. Zhaohui H, Yanli Z, Qin L, et al. Sensitive simultaneous determination of catechol and hydroquinone using a gold electrode modified with carbon nanofibers and gold nanoparticles. Microchim Acta. 2011;173:119-125. https://doi.org/10.1007/s00604-010-0530-y

20. Yanhui S, Ting Y, Xinfei Z, et al. Microsensor for hydroquinone and catechol based on poly (3,4-ethylenedioxidythiophene) modified carbon fiber electrode. Analyt Methods. 2016;8:886-892. https://doi.org/10.1039/C5AY02532J 
21. Ceren S, Gulsah C, Arzum E. Development of ionic liquid modified disposable graphite electrodes for label-free electrochemical detection of DNA hybridization related to Microcystis spp. Sensors. 2015;15:2273722749. https://doi.org/10.3390/s150922737

22. Xiaoying S, Song H, Linfang L, et al. Wei S. Sensitive electrochemical detection of hydroquinone with carbon ionogel electrode based on BMIMPF $_{6}$ J Electroanalyt Chem. 2011;651:94-99. http://dx.doi.org/10.1016/j.jelechem.2010.10.019

23. Wuxiang Z, Jianzhong Z, Zhongqiu L, et al. High sensitive simultaneous electrochemical determination of hydroquinone. Catechol and resorcinol based on carbon dots/reduced graphene oxide composite modified electrode. Analyt Methods. 2015;7:6089-6094. https://doi.org/10.1039/C5AY00848D

24. Arehalli S, Bahaddurghatta EKS. Electrosensitive determination of paracetamol using a poly(glycine) film coated graphite pencil electrode: A voltammetric study. Analyt Bioanal Electrochem. 2015;7:12- 21. https://doi.org/10.4172/2155-9872.1000260

25. Levent O, Mutlu S, Yücel S. Electrochemical preparation of a molecularly iprinted polypyrrole-modified pencil graphite electrode for determination of ascorbic acid. Sensors. 2008;8:5792-5805. https://doi.org/10.3390/s8095792 